\title{
The use of patient specific polyetheretherketone implants for reconstruction of maxillofacial deformities
}

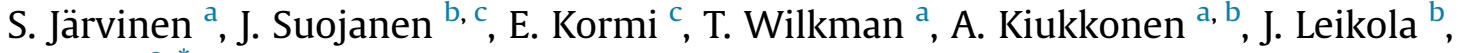 \\ P. Stoor ${ }^{\text {a, * }}$ \\ a Department of Oral and Maxillo-Facial Diseases at the University of Helsinki, Helsinki University Hospital, Haartmaninkatu 4E, 00029, HUS, Helsinki, \\ Finland \\ ${ }^{\mathrm{b}}$ Cleft Palate and Craniofacial Centre, Department of Plastic Surgery, Helsinki University Hospital, Topeliuksenkatu 5 (P.O. Box 266), 00029, HUS, Helsinki, \\ Finland \\ ${ }^{c}$ Päijät-Häme Joint Authority for Health and Wellbeing, Department of Oral and Maxillo-Facial Surgery, Finland
}

\section{A R T I C L E I N F O}

\section{Article history:}

Paper received 23 October 2018

Accepted 19 March 2019

Available online $\mathrm{xxx}$

\section{Keywords:}

Patient-specific implant

Custom implant

PEEK

Patient-specific modeling

Prostheses and implants

Deformity

\begin{abstract}
A B S T R A C T
Purpose: The aim of this study was to evaluate polyetheretherketone (PEEK) as a patient-specific implant (PSI) material in maxillofacial reconstructive surgery.

Materials and methods: The retrospective study included a cohort of 24 patients who underwent maxillofacial surgery using PEEK PSIs. Each patient underwent preoperative multislice computed tomography (CT) with $0^{\circ}$ tilt of gantry. Based on the CT scan, the PEEK PSIs were planned and manufactured using three-dimensional (3D) modeling and computer-aided design/computer-aided manufacturing (CAD/CAM) techniques. All procedures were performed under general anesthesia. Implants were placed intraorally, extraorally or through subciliary, transconjuctival or coronal incisions. Results: In 19 of 24 cases, the PEEK PSI fit well without adjustments. Although the fit to the surrounding bone was perfect in almost all of the cases, the outer contour of the PSI was modified in nine cases before fixation. However, intraoperative implant modification did not affect the infection rate. In two cases, postoperative wound dehiscence and infection needed additional treatment and healed without removal of the implants.

Conclusion: The follow-up data in this study showed good outcomes with reliable results for PSI made of PEEK in the maxillofacial region.
\end{abstract}

(c) 2019 European Association for Cranio-Maxillo-Facial Surgery. Published by Elsevier Ltd. All rights reserved.

\section{Introduction}

Many diseases and pathologies cause deformities and defects in the maxillofacial skeleton. These may cause malfunction and esthetic deformities, and therefore lead to a negative psychological impact (Scolozzi et al., 2007). Asymmetries and major underdevelopment of the maxillofacial skeleton are complex to reconstruct but necessary for esthetic and functional reasons. Many synthetic materials, such as titanium, alumina ceramics, porous polyethylene, and methyl methacrylate, have been used in maxillofacial

\footnotetext{
* Corresponding author. Department of Oral and Maxillo-Facial Diseases, Helsinki University Hospital, Finland Helsinki University Central Hospital, Haartmaninkatu 4E, P.O. Box 266, 00029, HUS, Helsinki, Finland.

E-mail addresses: patricia.stoor@hotmail.com, patricia.m.stoor@husmail.fi (P. Stoor).
}

reconstruction as alloplastic implants. The use of digitally designed patient-specific alloplastic implants has been reported to reduce time of surgery and is an effective technique in craniofacial reconstruction (Eppley et al., 2002).

In minor defects, autologous bone has also been used in maxillofacial reconstruction. It is relatively inexpensive compared to alloplastic materials, does not give rise to any immunogenic response, and integrates well biologically. However, the use of autologous bone is time-consuming, and there is additional morbidity due to the surgery needed for harvesting the auto transplant. The autologous bone graft may also have unpredictable resorption, leading to unstable long-term results.

Polyetheretherketone (PEEK) is a semicrystalline polyaromatic linear polymer. It is mechanically strong, nonallergenic, and nonmagnetic. PEEK has good biocompatibility and radiographic translucency (Nieminen et al., 2008). It has been used as an 
alloplastic biomaterial in craniofacial reconstructions (Scolozzi et al., 2007) and has been successfully used in orthopedic and spinal surgery (Kurtz and Devine, 2007) as well. PEEK implants provide permanent long-term results and are easily trimmed intraoperatively if needed. In this study, we report a series of 24 patients who underwent maxillofacial surgery with defects reconstructed using PEEK patient-specific implants (PSIs).

\section{Material and methods}

We report of retrospective cohort of 24 patients who underwent maxillofacial surgery using PEEK patient-specific implants (PSIs) between June of 2013 and November of 2018 at the Helsinki University Hospital and Päijät-Häme central hospital, Finland. The collected data included patient demographics, medical records, imaging studies and operative reports.

The lower facial esthetics and the shape of the mentolabial fold are determined by the position, shape and size of the chin (Naini and Gill, 2017). The draft plan was made at first by Dolphin Imaging Software. The McNamara analysis was used in the planning of the genioplasty (McNamara, 1984). In this method, the anatomic Frankfurt plane is determined, and the vertical perpendicular line is drawn through the Nasion-point. The antero-posterior position of the maxilla and the mandible is evaluated in relation to this vertical line. In the ideal situation, the A-point of the maxilla should be slightly farther than the vertical line, and the pogonion-point of the mandible should be slightly behind the vertical line (Proffit, 2013). The lower part of the face was vertically evaluated by quantifying the distance between the anterior nasal spine (ANS) and the Menton-point (Me) (Nanda, 2005). In the planning of the genioplasty, the antero-posterior location of the chin was evaluated in relation to the vertical line that was determined as in the McNamara analysis. The vertical facial height was measured by the distance between ANS and Me. The chin was moved forward and vertically positioned to match the ideal situation.

A preoperative multislice computed tomography (CT) with $0^{\circ}$ tilt of gantry was obtained from each patient. In general, a slice thickness of $1 \mathrm{~mm}$ is the minimum, but in thin bone areas such as the maxillary sinus anterior wall, $0.625 \mathrm{~mm}$ is recommended. Based on the CT scan, the PEEK PSIs were planned and manufactured in collaboration with a surgeon and an engineer using threedimensional (3D) modeling and computer-aided design/computeraided manufacturing (CAD/CAM) techniques. The full workflow is presented in detail in Fig. 1.

Individual patient characteristics can be seen in Tables 1 and 2 . In patients receiving a genioplasty, the indication was mandibular retrognathia with additional lip closure incompetence. In three cases (No. 5, 23 and 24), the mandibular reconstruction was achieved using three implants to correct a major asymmetry of the mandible and mentum. In four cases (No. 2, 8, 14 and 17), the reconstruction was achieved using two implants. Only one PSI was required to correct defects in the 17 other cases. The PEEK implants were sent to the hospital and sterilized prior to surgery. In this study, two PSIs (No. 7 and 19) were manufactured by DePuy Synthes, Switzerland and 22 PSIs by Planmeca Ltd, Finland.

All procedures were performed under general anesthesia. In 16 cases, implants were placed intraorally. Two of these implants were placed to the zygomatic bone and 10 to the mandible. Subciliary, transconjuctival or coronal approaches to the orbital bones were used in 7 cases. One genioplasty was performed through an extraoral incision. If the patient had had previous procedures, earlier implants were removed during the surgery. The PEEK implant was trimmed with a cutting burr if it was needed to be reshaped or if the esthetic result was not satisfying. PEEK PSIs were fixed to the surrounding bone with Matrix Midface or Matrix Orthognathic titanium screws (DePuy Synthes).

\section{Results}

The study included 24 patients ( 15 female and 9 male) with an age range from 16 to 72 years, the mean age being 30.8 years. The follow-up period ranged from 2 to 63 months, the average followup period being 16.2 months. Patients' demographic and pathological features are presented in Table 1 individually for each patient, and the results are detailed in Table 2. Briefly, defects in the mandible accounted for 14 , the zygomatic area for 3 , and the orbital area for 7 of the cases that were corrected with PEEK PSI. In 19 of 24 cases, the PEEK PSI fit well without adjustments. One of the two PSIs (No. 19) that did not fit well onto the underlying bone was trimmed. In the other case (No. 1), a small gap between the implant and the mandible was patched with cancelous bone. Although the fit to the surrounding bone was perfect in 19 of 24 cases, the outer contour of the PSI was modified in 9 cases before fixation. In addition, one single orbital floor implant (No. 20) was modified due to poor ocular globe position.

All patients received perioperative intravenous antibiotics. Cefuroxime $1.5 \mathrm{~g}$ was administered in 20 cases and in four cases the patient received an additional $500 \mathrm{mg}$ of metronidazole. Three patients received ampicillin $2 \mathrm{~g}$ and one patient clindamycin $600 \mathrm{mg}$. In seven cases, the PSI was immersed in antibiotic solution before fitting and fixation (two cases of ciprofloxacin and five cases of cefuroxime). Antibiotics were also prescribed for 21 patients postoperatively for 7-14 days. Cephalexin $500 \mathrm{mg}$ was prescribed in 10 , cefuroxime $500 \mathrm{mg}$ in one case, phenoxymethylpenicillin 1 million IU in four cases, clindamycin $300 \mathrm{mg}$ in two cases and amoxicillin $500 \mathrm{mg}$ in four cases. Two of the patients receiving amoxicillin had also metronidazole $400 \mathrm{mg}$ prescribed. In three cases (No. 10, 13 and 14), the patients received antibiotics only for 2 days postoperatively and were given intravenous cefuroxime and metronidazole during the hospital stay.

Wound dehiscence occurred in two cases, and in one of these the implant was exposed. Only one of the dehiscence wounds was clinically infected (No. 13) and was treated with resuturation and antibiotics 20 days after surgery (early infection, defined as occurrence between weeks 0 and 4 postoperatively in this study). In the other case (No. 7), the wound dehiscence did not show any clear marks of infection, even though the implant was exposed intraorally, and the patient was followed up for over 10 months after surgery. After 10 months of follow-up, the implant at the angle of the mandible was still exposed at the superior and anterior border of the ramus, and the patient underwent a second surgical procedure in which the PSI was trimmed to become lower and smoother buccally. The wound healed well after the implant modification, but an intra oral fistula re-occurred without evidence of a clinical infection. Because of the fistula, the patient underwent yet another surgical procedure in which the fistula was removed and granulation tissue was purified around the PSI. No other early or any late infections (defined as occurrence after 4 weeks postoperatively) occurred.

In one case (No. 2), 9 months after the first operation, a reoperation was needed due to major weight loss that led to the asymmetry of mandibular body becoming clinically visible. The implant correcting the defect of the mandibular symphysis area was replaced with two new implants that corrected the asymmetry more widely. Neither wound dehiscence nor infections occurred after the operations in this patient.

Minor paresthesia was present in six cases (No. 2, 3, 4, 6, 14 and 22). In one case (No. 8), the PSIs were placed on zygomatic bones and lateral orbital rims. After this, the patient experienced transient 


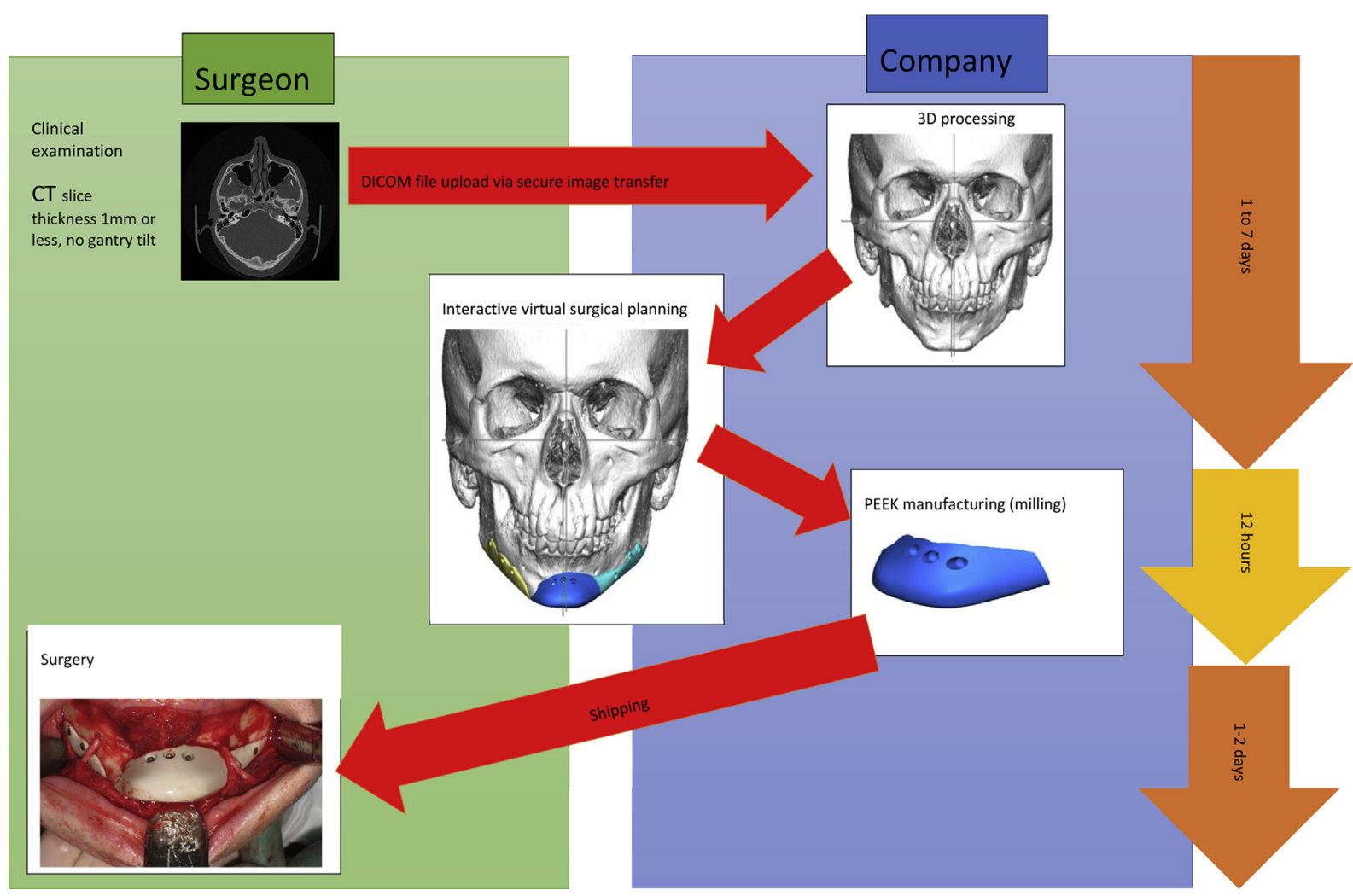

Fig. 1. Workflow for the design and use of polyetheretherketone (PEEK) patient-specific implants (PSI) in maxillofacial surgery. The clinician part of the process is presented on the left (green). The amount of asymmetry to be corrected is decided based on clinical examination and radiographic imaging. The computer-aided design/computer aided manufacturing (CAD/CAM) process in the company is presented on the right (blue). The CAM part itself is rapid and is only rarely the reason for delays in the treatment. In this chart, the workflow of patient No. 24 in presented. The mandible corpus asymmetry is corrected using three PSIs. This puzzle-type design of the PSI helps in placing the implants without nerve damage through an intraoral approach. All implants are self-positioning and/or interlocking onto mandible contours, and no drill guide was needed in this case.

Table 1

Patient characteristic.

\begin{tabular}{|c|c|c|c|c|}
\hline Patient No. & Sex & Age & Diagnosis & Previous operations \\
\hline 1 & $\mathrm{~F}$ & 23 & Other specified jaw size anomalies & Rapid expansion of maxilla, Bimaxillary osteotomy \\
\hline 2 & $\mathrm{~F}$ & 33 & Mandibular retrognathism & Bimaxillary osteotomy \\
\hline 3 & M & 19 & Mandibular retrognathism & None \\
\hline 4 & $\mathrm{~F}$ & 22 & Juvenile rheumatoid polyarthritis & None \\
\hline 5 & M & 35 & Mandibular asymmetry & Bimaxillary osteotomy and genioplasty \\
\hline 6 & $\mathrm{~F}$ & 20 & Mandibular asymmetry & None \\
\hline 7 & $\mathrm{~F}$ & 21 & Goldenhar syndrome & $\begin{array}{l}\text { Reconstruction of mandible with costochondral graft, bimaxillary osteotomy, } \\
\text { free abdominal fat transfer to mandible angle }\end{array}$ \\
\hline 8 & M & 23 & $\begin{array}{l}\text { Other malformation syndrome } \\
\text { predominantly affecting facial appearance }\end{array}$ & $\begin{array}{l}\text { Ear reconstruction, Bimaxillary osteotomy (at the same time with } \\
\text { PEEK PSI placement) }\end{array}$ \\
\hline 9 & $\mathrm{~F}$ & 26 & Mandibular retrognathism & None \\
\hline 10 & $\mathrm{~F}$ & 24 & Orbital deformity (after trauma) & Reconstruction of multiple skull defects after trauma \\
\hline 11 & M & 72 & Fracture of orbital floor & $\begin{array}{l}\text { Reconstruction of orbital wall, reduction and osteosynthesis of } \\
\text { zygomaticomaxillary fracture }\end{array}$ \\
\hline 12 & $\mathrm{~F}$ & 49 & Mandibular retrognathism & Prosthetic replacements of mandibular joints \\
\hline 13 & M & 20 & Apert syndrome & Cranioplasty (twice), Le Fort III osteotomy \\
\hline 14 & M & 21 & Crouzon syndrome & Cranioplasty (three times), maxillary distraction \\
\hline 15 & $\mathrm{~F}$ & 20 & Hemifacial microsomia & None \\
\hline 16 & $\mathrm{~F}$ & 49 & TMD, Openbite & Bimaxillary osteotomy \\
\hline 17 & $\mathrm{~F}$ & 22 & Achondroplasia, Maxillary retrognathism & Bimaxillary osteotomy \\
\hline 18 & $\mathrm{~F}$ & 31 & Asymmetry & Recection of mandible, prosthetic replacement of mandibular joint \\
\hline 19 & $\mathrm{~F}$ & 46 & $\begin{array}{l}\text { Malignant neoplasm of choroid, Acquired } \\
\text { absence of part of orbital bones }\end{array}$ & Enucleation of corpus ciliare \\
\hline 20 & $\mathrm{~F}$ & 16 & Fracture of orbital floor & Reconstruction of middle facial fractures \\
\hline 21 & $\mathrm{~F}$ & 72 & $\begin{array}{l}\text { Malignant neoplasm of choroid, Acquired } \\
\text { absence of part of orbital bones }\end{array}$ & Enucleation \\
\hline 22 & M & 22 & Mandibular retrognathism & None \\
\hline 23 & M & 31 & Mandibular retrognathism & Bimaxillary osteotomy \\
\hline 24 & M & 22 & Juvenile rheumatoid polyarthritis & None \\
\hline
\end{tabular}

Abbreviations in the table are No, number; F, female; M, male; PEEK, polyetheretherketone; PSI, patient specific implant; TMD, temporomandibular disorders. 
Table 2

PEEK PSI characteristics and results.

\begin{tabular}{|c|c|c|c|c|c|c|}
\hline Patient No. & $\begin{array}{l}\text { PEEK PSI } \\
\text { Manufacturer }\end{array}$ & $\begin{array}{l}\text { Number } \\
\text { of PSI }\end{array}$ & PSI site & Access & Complications & $\begin{array}{l}\text { Follow-up } \\
\text { (mo) }\end{array}$ \\
\hline 1 & Planmeca & 1 & Symphysis of mandible & Intraoral & None & 19 \\
\hline 2 & Planmeca & 1,2 & Symphysis and body of mandible & Intraoral & Minor paresthesia & 10 \\
\hline 3 & Planmeca & 1 & Symphysis of mandible & Intraoral & Minor paresthesia & 3 \\
\hline 4 & Planmeca & 1 & Symphysis of mandible & Intraoral & Minor paresthesia & 23 \\
\hline 5 & Planmeca & 3 & Symphysis and body of mandible & Intraoral & None & 10 \\
\hline 6 & Planmeca & 1 & Symphysis of mandible & Intraoral & Minor paresthesia & 2 \\
\hline 7 & Synthes & 1 & $\begin{array}{l}\text { Angle of mandible } \\
\text { (costochondral graft) }\end{array}$ & Intraoral and extraoral & Prolonged wound dehiscence & 51 \\
\hline 8 & Planmeca & 2 & $\begin{array}{l}\text { Zygomatic bones and } \\
\text { lateral orbital rims }\end{array}$ & $\begin{array}{l}\text { Intraoral and lateral canthotomy } \\
\text { and conjuctival }\end{array}$ & $\begin{array}{l}\text { Transient facial paralysis of the } \\
\text { zygomatic branch of the } \\
\text { facial nerve }\end{array}$ & 21 \\
\hline 9 & Planmeca & 1 & Symphysis of mandible & Intraoral & None & 4 \\
\hline 10 & Planmeca & 1 & Orbital bones & Subciliary & None & 26 \\
\hline 11 & Planmeca & 1 & Orbital floor & $\begin{array}{l}\text { Transconjuctival and lateral } \\
\text { canthotomy }\end{array}$ & None & 11 \\
\hline 12 & Planmeca & 1 & Symphysis of mandible & Extraoral & None & 11 \\
\hline 13 & Planmeca & 1 & Lateral orbital rim & Intraoral and subciliary & Early infection & 8 \\
\hline 14 & Planmeca & 2 & Lateral orbital rim & Intraoral & Minor paresthesia & 63 \\
\hline 15 & Planmeca & 1 & Symphysis of mandible & Intraoral & None & 3 \\
\hline 16 & Planmeca & 1 & Symphysis of mandible & Intraoral & None & 28 \\
\hline 17 & Planmeca & 2 & Zygomatic bone & Intraoral & None & 10 \\
\hline 18 & Planmeca & 1 & Zygomatic bone & Intraoral & None & 6 \\
\hline 19 & Synthes & 1 & Orbital floor & Transconjuctival & None & 27 \\
\hline 20 & Planmeca & 1 & Orbital floor and infraorbital rim & $\begin{array}{l}\text { Transconjuctival and lateral } \\
\text { canthotomy }\end{array}$ & None & 42 \\
\hline 21 & Planmeca & 1 & Orbital floor & Subciliary & None & 3 \\
\hline 22 & Planmeca & 1 & Symphysis of mandible & Intraoral & Minor paresthesia & 3 \\
\hline 23 & Planmeca & 3 & Symphysis and body of mandible & Intraoral & None & 2 \\
\hline 24 & Planmeca & 3 & Symphysis and body of mandible & Intraoral & None & 2 \\
\hline
\end{tabular}

Abbreviations in the table are No, number; PEEK, polyetheretherketone; PSI, patient specific implant; mo, months.

facial paralysis of the zygomatic branch of the facial nerve that occurred 2 weeks after surgery and that was probably caused by postoperative swelling.

\section{Discussion}

PEEK has many advantages compared with other alloplastic implant materials. PEEK has radiographic translucency (Nieminen et al., 2008) and produces no artifacts on radiographic imaging. For example, titanium does not have this feature and may cause diagnostic difficulties. PEEK is also nonallergenic and nonmagnetic and does not undergo exothermic reactions like methyl methacrylate does (Shah et al., 2014). Furthermore, PEEK is comparable to cortical bone regarding its elasticity (Lethaus et al., 2012), unlike some other alloplastic materials, such as titanium. The disadvantage of pure PEEK is that it has no bioactive potential (Lethaus et al., 2011). None of the alloplastic materials used have been reported being superior to others. When using an alloplastic implant, donor site morbidity is avoided and operative time is reduced, especially when the PSI does not need to be modified intraoperatively.

In the literature, there are divergent study conclusions regarding whether intraoperative antibiotics have had (Hey et al., 2017) or have not had (Suh et al., 2015) an effect on infection rates in orthopedic surgery. In this study, the use of intraoperative antibiotics with the PSIs had no clear effect on the amount of infections. Intraoperative implant modification shows no evident influence on the infection rate either. In the case of patient No. 13, whose infection occurred at an implant located at the lateral orbital rim, the reason for the infection remains uncertain. The implant was placed through intraoral and subciliary incisions. This patient had no further prescription for antibiotics after the 2 days of hospital stay. However, it may be safer for patients if simultaneous intraoral and extraoral approaches can be avoided.
The reason for wound dehiscence without clear signs of acute infection seems clear, as in patient No. 7: an overly large PSI volume with a sharp edge was attempted to be covered with a relatively thin mucous membrane intraorally. The implant volume and shape in different anatomical locations should always be evaluated precisely, not only to achieve good esthetic and functional results but also to ensure that the surrounding soft tissue can adapt to it.

Regarding the planning of PEEK PSIs used for genioplasty, we found that the preoperative CT scan needs to be precisely taken with the mandible in the condylar position. Patients easily protrude their mandible habitually to compensate for the retrognathic or asymmetric mandible. An occlusional index with the mandible in the condylar position should thus be used during the CT scan so as to be able to provide a fully symmetric and anatomical reconstruction of the lower jaw with the PSI. Cone beam computed tomography (CBCT) can be also used for the design; however, in this study, all patients were examined with conventional CT. If CBCT is used, it must be noted that a sufficiently large field of view should be selected to permit adequate planning and evaluation of symmetry.

Our infection rate was $8.3 \%$, which is comparable to that of other craniofacial PEEK PSI case series. Alonso-Rodriguez et al. (2015) reported a series of 14 cases with an infection rate of $14.3 \%$, and Rosenthal et al. (2014) published a study of 65 cases in with an infection rate of $7.7 \%$.

\section{Conclusion}

In conclusion, the follow-up data in this study showed good outcomes with reliable results for PSI made of PEEK in the maxillofacial region. 


\section{Acknowledgements}

We would like to thank Emil Aaltonen Foundation, FinnishNorwegian Medical Foundation, The Finnish Medical Foundation, Paulo Foundation, and Helsinki University Hospital Funds for financial support to this study.

\section{References}

Alonso-Rodriguez E, Cebrián JL, Nieto MJ, Del Castillo JL, Hernández-Godoy J, Burgueño M: Polyetheretherketone custom-made implants for craniofacial defects: report of 14 cases and review of the literature. J Craniomaxillofac Surg 43(7): 1232-1238, 2015

Eppley BL, Kilgo M, Coleman JJ: Cranial reconstruction with computer-generated hard-tissue replacement patient-matched implants: indications, surgical technique, and long-term follow-up. Plast Reconstr Surg 109(3): 864-871, 2002

Hey HW, Thiam DW, Koh ZS, Thambiah JS, Kumar N, Lau LL, et al: Is intraoperative local vancomycin powder the answer to surgical site infections in spine surgery? Spine 42(4): 267-274, 2017

Kurtz SM, Devine JN: PEEK biomaterials in trauma, orthopedic, and spinal implants. Biomaterials 28(32): 4845-4869, 2007

Lethaus B, Ter Laak MP Laeven P. Beerens M, Koper D, Poukens J et al: A treatment algorithm for patients with large skull bone defects and first results. J Craniomaxillofac Surg 39(6): 435-440, 2011
Lethaus B, Safi Y, ter Laak-Poort M, Kloss-Brandstätter A, Banki F, Robbenmenke C, et al: Cranioplasty with customized titanium and PEEK implants in a mechanical stress model. J Neurotrauma 29(6): 1077-1083, 2012

McNamara Jr JA: Dentofacial adaptations in adult patients following functional regulator therapy. Am J Orthod 85(1): 57-71, 1984

Naini FB, Gill SG: Orthognathic surgery. Osseus Genioplasty: 571-580, 2017

Nanda R: Biomechanics and esthetic strategies in clinical orthodontics. Philadelphia, PA, USA: Elsevier Inc., 64, 2005

Nieminen T, Kallela I, Wuolijoki E, Kainulainen H, Hiidenheimo I, Rantala I: Amorphous and crystalline polyetheretherketone: mechanical properties and tissue reactions during a 3-year follow-up. J Biomed Mater Res A 84(2): 377-383, 2008

Proffit WR: Contemporary orthodontics, 5th ed. St Louis, Missouri, USA: Mosby Inc., an imprint of Elsevier Inc.,, 194, 2013

Rosenthal G, Ng I, Moscovici S, Lee KK, Lay T, Martin C, et al: Polyetheretherketone implants for the repair of large cranial defects: a 3-center experience. Neurosurgery 75(5): 523-529, 2014

Scolozzi P, Martinez A, Jaques B: Complex orbito-fronto-temporal reconstruction using computer-designed PEEK implant. J Craniofac Surg (1): 224-228, 2007

Shah AM, Jung H, Skirboll S: Materials used in cranioplasty: a history and analysis. Neurosurg Focus 36(4): E19, 2014

Suh Bo-Kyung, Moon Seong-Hwan, Kim Tae-Hwan, Oh Jae Keun, Kwon Yong Shin, Park Jung-Seob, et al: Efficacy of antibiotics sprayed into surgical site for prevention of the contamination in the spinal surgery. Asian Spine J 9(4): $517-521,2015$ 